\title{
The Nature of Inertial and Vacuum Gravitational Field
}

\author{
Ning Wu \\ Center for Theoretical Study, Beijing, China \\ Email: wuning_88@163.com
}

How to cite this paper: $\mathrm{Wu}, \mathrm{N}$. (2016) The Nature of Inertial and Vacuum Gravitational Field. Journal of Modern Physics, 7, 21262134.

http://dx.doi.org/10.4236/jmp.2016.715185

Received: October 20, 2016

Accepted: November 8, 2016

Published: November 11, 2016

Copyright (๑) 2016 by author and Scientific Research Publishing Inc. This work is licensed under the Creative Commons Attribution International License (CC BY 4.0).

http://creativecommons.org/licenses/by/4.0/ (c) (i) Open Access

\begin{abstract}
The uniformly accelerated motion is studied in the framework of gauge theory of gravity. It is found that, when an inertial reference system is transformed into a uniformly accelerated system by a local gravitational gauge transformation, a nontrivial gravitational gauge field appears. If there is a mass point in the new reference frame, there will be a non-trivial gravitational force acting on it. The nature and the characteristic of this new force are completely the same as those of the traditional inertial force. This new gravitational force is considered to be the inertial force. Therefore, the nature of inertial force is gravity, which is the basic idea of the equivalence principle.
\end{abstract}

\section{Keywords}

Lorentz Transformation, Gravitational Gauge Transformation, Inertial Force, Gauge Theory of Gravity, Gravitational Gauge Field

\section{Introduction}

According to the Newton's second law of motion, when there is an external force $F$ acted on a mass point $m$, the mass point will be uniformly accelerated along the direction of the external force. In this paper, we will study another problem: if we sit in the center of mass system of the mass point $m$, what will we discover? This question should be answered by an astronaut. When an astronaut sits in a rocket which is launching, what will he discover? He will discover that there is a huge force acting on him which is many times larger than his weight. Where does the extra force come from? What is the nature of the extra force? It is known that there are four kinds of fundamental interactions in nature. They are electromagnetic interaction, weak interaction, strong interaction, and gravitational interaction. Firstly, this force cannot be weak interaction or 
strong interaction, for these two kinds of interactions are short range. Secondly, this force cannot be electromagnetic force, for the astronaut carries no electric charge. Therefore, the only possibility left is that it is a gravitational force. But this force cannot be explained in either Newton's classical theory of gravity [1] [2] or in Einstein's general theory of relativity [3] [4] [5]. According to both Newton's classical theory of gravity and Einstein's general theory of relativity, in order to produce so strong gravitational force, there should a massive object nearby whose mass is much larger than that of earth. But it is obvious that there does not exist such an object near the rocket. So, according to both Newton's classical theory of gravity and Einstein's general theory of relativity, the possibility that this force is gravitational force seems to be ruled out. Now, we return back to the former question: what is the nature of the force? In classical mechanics, this force is called inertial force, or fictitious force [1] [2]. Because physicists cannot find the origin of this force, it is generally believed that the inertial force does not actually exist in nature; it is only a fictitious force. However, this point of view does not agree with facts, for the astronaut in a rocket feels that this great force does really exist. In other words, the existence of this force is based on the feeling of the astronaut, not based on our hypothesis.

In this paper, the problem of inertial force is studies in the framework of quantum gauge theory of gravity. Quantum Gauge Theory of Gravity (QGTG) is proposed in 2001 [6] [7] [8] [9]. The motivation to propose QGTG is try to unify general relativity with quantum theory in the framework of gauge field theory. In 2003, Quantum Gauge General Relativity (QGGR) is proposed in the framework of QGTG [6]-[12]. Unlike Einstein's general theory of relativity, the cornerstone of QGGR is the gauge principle, not the principle of equivalence, which will cause far-reaching influence to the theory of gravity. In QGGR, the field equation of gravitational gauge field is just the Einstein's field equation, and in classical level, QGGR returns to Einstein's general relativity [13]. The field equation of gravitational gauge field in QGGR is equivalent to the Einstein's field equation in general relativity, so two equations have the same solutions, though mathematical expressions of the two equations are completely different. For classical tests of gravity, QGGR gives out the same theoretical predictions as those of GR [14] [15] [16], and for non-relativistic problems, QGGR can return to Newton's classical theory of gravity [15]. Based on the coupling between the spin of a particle and gravito electromagnetic field, the equation of motion of spin can be obtained in QGGR. In post Newtonian approximations, this equation of motion of spin gives out the same results as those of GR [16]. The equation of motion of a spinning test particle in gravitational field can also be obtained [17]. It's found that this motion deviates from traditional geodesic curve, and the deviation effect is detectable [18], which is a new classical test of gravity theory. QGGR is a perturbatively renormalizable quantum theory, so based on it, quantum effects of gravity [19] [20] [21] [22] and gravitational interactions of some basic quantum fields [23] [24] can be explored. Unification of fundamental interactions including gravity can be fulfilled in a semi-direct product gauge group [25] [26] [27] [28]. If we use the mass generation mechanism which is proposed in literature 
[29] [30], we can propose a new theory on gravity which contains massive graviton and the introduction of massive graviton does not affect the strict local gravitational gauge symmetry of the action and does not affect the traditional long-range gravitational force [31]. The existence of massive graviton will help us to understand the possible origin of dark matter.

In the literature [32], the problem of the change of space-time structure under Lorentz transformation is studied. In that paper, only the Lorentz transformation of uniform motion in a straight line is studied. If the motion is constantly accelerated motion, what will happen? In this paper, the gravitational field in a local constantly accelerated reference is studied. In Section 2, a simple introduction to the gauge theory of gravity is given. The gravitational field and the field strength of gravitational field in a constantly accelerated reference are calculated in Section 3. The gravitational force on a mass point in that local reference is studied in Section 4. Finally, some discussions and comments are given.

\section{Quantum Gauge Theory of Gravity}

A simple introduction on the quantum gauge theory of gravity is given in this chapter. Details on this theory can be found in literatures [6]-[12]. In quantum gauge theory of gravity, the most fundamental physical quantity is gravitational gauge field $C_{\mu}(x)$, which is a vector in the corresponding Lie algebra. $C_{\mu}(x)$ can be expanded as

$$
C_{\mu}(x)=C_{\mu}^{\alpha}(x) \hat{P}_{\alpha},(\mu, \alpha=0,1,2,3)
$$

where $C_{\mu}^{\alpha}(x)$ is the component field and $\hat{P}_{\alpha}=-i \frac{\partial}{\partial x^{\alpha}}$ is the generator of global gravitational gauge group. The gravitational gauge covariant derivative is given by

$$
D_{\mu}=\partial_{\mu}-i g C_{\mu}(x)=G_{\mu}^{\alpha}(x) \partial_{\alpha},
$$

where $g$ is the gravitational coupling constant and matrix $G$ is given by

$$
G=\left(G_{\mu}^{\alpha}\right)=\left(\delta_{\mu}^{\alpha}-g C_{\mu}^{\alpha}\right) .
$$

Its inverse matrix is

$$
G^{-1}=\frac{1}{I-g C}=\left(G_{\alpha}^{-1 \mu}\right) .
$$

Using matrix $G$ and $G^{-1}$, we can define two important composite operators

$$
\begin{aligned}
& g^{\alpha \beta}=\eta^{\mu v} G_{\mu}^{\alpha} G_{v}^{\beta}, \\
& g_{\alpha \beta}=\eta_{\mu \nu} G_{\alpha}^{-1 \mu} G_{\beta}^{-1 v} .
\end{aligned}
$$

Quantum gauge theory of gravity is formulated in absolute space-time [13] [33]. Therefore, in quantum gauge theory of gravity, space-time is always flat and space-time metric is always Minkowski metric, so $g^{\alpha \beta}$ and $g_{\alpha \beta}$ are no longer space-time metric. They are only two composite operators which consist of gravitational gauge field. Einstein's general relativity is formulated in physical space-time, and $g^{\alpha \beta}$ and $g_{\alpha \beta}$ are metric of physical space-time. 
The field strength of gravitational gauge field is defined by

$$
F_{\mu v}(x) \triangleq \frac{1}{-i g}\left[D_{\mu}, D_{v}\right]=F_{\mu \nu}^{\alpha}(x) \cdot \hat{P}_{\alpha}
$$

where

$$
F_{\mu v}^{\alpha}=G_{\mu}^{\beta} \partial_{\beta} C_{v}^{\alpha}-G_{v}^{\beta} \partial_{\beta} C_{\mu}^{\alpha}
$$

The Lagrangian of the quantum gauge theory of gravity is selected to be

$$
\mathcal{L}=\left(\operatorname{det} G^{-1}\right) \mathcal{L}_{0}
$$

where

$$
\mathcal{L}_{0}=-\frac{1}{16} \eta^{\mu \rho} \eta^{\nu \sigma} g_{\alpha \beta} F_{\mu \nu}^{\alpha} F_{\rho \sigma}^{\beta}-\frac{1}{8} \eta^{\mu \rho} G_{\beta}^{-1 v} G_{\alpha}^{-1 \sigma} F_{\mu \nu}^{\alpha} F_{\rho \sigma}^{\beta}+\frac{1}{4} \eta^{\mu \rho} G_{\alpha}^{-1 v} G_{\beta}^{-1 \sigma} F_{\mu \nu}^{\alpha} F_{\rho \sigma}^{\beta} .
$$

Its space-time integration gives out the action of the system

$$
S=\int \mathrm{d}^{4} x \mathcal{L}
$$

Under gravitational gauge transformations [6] [7] [8] [9], the gauge transformation of space-time coordinates is

$$
x^{\mu} \rightarrow y^{\mu}=\left(\hat{U}_{\epsilon}(x) x^{\mu}\right)=x^{\mu}-\epsilon^{\mu} .
$$

The gauge transformation of gravitational gauge field is

$$
C_{\mu}(x) \rightarrow C_{\mu}^{\prime}(x)=\hat{U}_{\epsilon}(x) C_{\mu}(x) \hat{U}_{\epsilon}^{-1}(x)+\frac{i}{g} \hat{U}_{\epsilon}(x)\left(\partial_{\mu} \hat{U}_{\epsilon}^{-1}(x)\right) .
$$

Using Equation (1), the above relation can be changed into

$$
C_{\mu}^{\alpha}(x) \rightarrow C_{\mu}^{\alpha}(x)=\Lambda_{\beta}^{\alpha}\left(\hat{U}_{\epsilon}(x) C_{\mu}^{\beta}(x)\right)-\frac{1}{g}\left(\hat{U}_{\epsilon}(x) \partial_{\mu} \epsilon^{\alpha}(f(x))\right),
$$

where $f(x)$ is a function of space-time coordinates which satisfy

$$
\left(\hat{U}_{\epsilon}(x) f(x)\right)=x,
$$

and $\Lambda_{\beta}^{\alpha}$ is defined by

$$
\Lambda_{\beta}^{\alpha}=\frac{\partial x^{\alpha}}{\partial(x-\epsilon(x))^{\beta}}=\frac{\partial x^{\alpha}}{\partial y^{\beta}}
$$

\section{Uniformly Accelerated Motion}

Now, let's study the Lorentz transformation of $x^{\alpha} \rightarrow y^{\alpha}=\Lambda_{\beta}^{\alpha} x^{\beta}$. The gravitational gauge transformation of gravitational gauge field itself under Lorentz transformation is given by relation (28) in literature [32]:

$$
C_{\mu}^{\alpha}(x) \rightarrow C_{\mu}^{\prime \alpha}(x)=\Lambda_{\beta}^{\alpha}\left(\hat{U}_{\epsilon} C_{\mu}^{\beta}(x)\right)-\frac{1}{g}\left(\Lambda_{\mu}^{\alpha}-\delta_{\mu}^{\alpha}\right),
$$

where $C_{\mu}^{\alpha}(x)$ is the gravitational gauge field before Lorentz transformation, and $C_{\mu}^{\alpha}(x)$ is the gravitational gauge field after Lorentz transformation. Suppose that the 
gravitational gauge field vanishes before Lorentz transformation, that is

$$
C_{\mu}^{\alpha}(x)=0 .
$$

The reference after Lorentz transformation is a moving reference, which is denoted by $\Sigma$. The gravitational gauge field $C_{\mu}^{\alpha}(x)$ in the moving reference $\Sigma$ is given by (17). That is

$$
C_{\mu}^{\prime \alpha}(x)=-\frac{1}{g}\left(\Lambda_{\mu}^{\alpha}-\delta_{\mu}^{\alpha}\right)
$$

By using (3) and (4), the following two relations can be obtained

$$
\begin{gathered}
G_{\mu}^{\prime \alpha}(x)=\delta_{\mu}^{\alpha}-g C_{\mu}^{\prime \alpha}(x)=\Lambda_{\mu}^{-1 \alpha}=\frac{\partial x^{\alpha}}{\partial y^{\mu}}, \\
G_{\alpha}^{\prime-1 \mu}(x)=\Lambda_{\alpha}^{\mu}=\frac{\partial y^{\mu}}{\partial x^{\alpha}} .
\end{gathered}
$$

For the sake of convenience, we suppose that the Lorentz transformation is along the direction of $\mathrm{X}$ axis. In this case, we have

$$
\begin{aligned}
& \frac{\partial y^{\mu}}{\partial x^{\alpha}}=\left(\begin{array}{cccc}
\gamma & \gamma v & 0 & 0 \\
\gamma \nu & \gamma & 0 & 0 \\
0 & 0 & 1 & 0 \\
0 & 0 & 0 & 1
\end{array}\right), \\
& \frac{\partial x^{\alpha}}{\partial y^{\mu}}=\left(\begin{array}{cccc}
\gamma & -\gamma \nu & 0 & 0 \\
-\gamma \nu & \gamma & 0 & 0 \\
0 & 0 & 1 & 0 \\
0 & 0 & 0 & 1
\end{array}\right),
\end{aligned}
$$

where $v$ is the velocity of the boost, and $\gamma=\frac{1}{\sqrt{1-v^{2}}}$.

Now, let's study the uniformly accelerated motion. In this case,

$$
v=a t .
$$

In this case, the moving reference $\Sigma$ is a uniformly accelerated reference. The gravitational gauge field in the moving reference $\Sigma$ is

$$
\begin{aligned}
& G_{\mu}^{\prime \alpha}(x)=\left(\begin{array}{cccc}
\gamma & -\gamma \nu & 0 & 0 \\
-\gamma \nu & \gamma & 0 & 0 \\
0 & 0 & 1 & 0 \\
0 & 0 & 0 & 1
\end{array}\right), \\
& G_{\alpha}^{\prime-1 \mu}(x)=\left(\begin{array}{cccc}
\gamma & \gamma \nu & 0 & 0 \\
\gamma \nu & \gamma & 0 & 0 \\
0 & 0 & 1 & 0 \\
0 & 0 & 0 & 1
\end{array}\right) .
\end{aligned}
$$

The field strength of gravitational gauge field is given by (8), that is

$$
-g F_{\mu \nu}^{\prime \alpha}=D_{\mu}^{\prime} G_{v}^{\prime \alpha}-D_{\nu}^{\prime} G_{\mu}^{\prime \alpha} \text {. }
$$


It is found that all components of $g F_{\mu v}^{\prime \alpha}$ vanish, except the following one

$$
g F_{t x}^{\prime t}=-g F_{x t}^{\prime t}=\gamma^{2} a,
$$

which corresponds to the following gravito-electric field

$$
g E_{x}^{t}=g F_{t x}^{\prime t}=\gamma^{2} a .
$$

So, there is a Newtonian gravitational term in the moving reference $\Sigma$, whose direction is along positive $\mathrm{X}$ axis, and whose magnitude is $\gamma^{2} a$.

\section{Gravitational Forc}

Through the study in the above chapter, we found that there exists non-trivial gravitational field in the uniformly accelerated reference $\Sigma$. Next, we will calculate the gravitational force on a mass point in the reference $\Sigma$. The gravitational force on a mass point with mass $m$ in the reference $\Sigma$ is given by

$$
f^{\mu}=m g \eta^{\mu v} g_{\alpha \beta}^{\prime} G_{\sigma}^{\prime-1 \lambda} F_{v \lambda}^{\prime \alpha} \frac{\mathrm{d} x^{\beta}}{\mathrm{d} \tau} \frac{\mathrm{d} x^{\sigma}}{\mathrm{d} \tau}
$$

Suppose that the mass point is moving along the $\mathrm{X}$ axis, that is

$$
\frac{\mathrm{d} x^{\alpha}}{\mathrm{d} \tau}=\left(\gamma_{u} ; \gamma_{u} u, 0,0\right)
$$

Please remember that the velocity $\frac{\mathrm{d} x^{\alpha}}{\mathrm{d} \tau}$ is the velocity of mass point in the absolute space-time [32] [33]. The velocity of mass point in the moving reference $\Sigma$ is $G_{\alpha}^{\prime-1 \mu} \frac{\mathrm{d} x^{\alpha}}{\mathrm{d} \tau}$. Substitute (6), (26), (28) and (31) into (30), we will obtain the gravitational force on the mass point

$$
\begin{gathered}
f^{t}=m a \gamma^{3} \gamma_{u}^{2}(u+v), \\
f^{x}=\operatorname{ma\gamma }^{3} \gamma_{u}^{2}(1+u v), \\
f^{y}=f^{z}=0 .
\end{gathered}
$$

In the above relation, $\gamma \gamma_{u}(u+v)$ is the relative velocity of mass point $\mathrm{m}$ in the moving reference $\Sigma$. If we suppose that the mass point $\mathrm{m}$ is at rest in the moving reference $\Sigma$, that is

$$
\gamma \gamma_{u}(u+v)=0
$$

In this case, $f^{t}$ vanishes, and

$$
f^{x}=\operatorname{mar}^{5}\left(1-v^{2}\right)=m a \gamma^{3}
$$

It means that the mass point rested in the accelerated reference $\Sigma$ feels a gravitational force with the magnitudes of $\gamma^{3} m a \cong m a$. From the point of view of boost transformation, the mass point is boosted along the positive $\mathrm{X}$ axis. But from the point of view of coordinate transformation, the coordinate system is moving along the negative $\mathrm{X}$ axis. Therefore, when the reference $\Sigma$ is accelerated along the negative $\mathrm{X}$ axis, the mass point 
$\mathrm{m}$ rested in the reference $\Sigma$ will feel a gravitational force with the magnitude of ma and with the direction of positive $\mathrm{X}$ axis. This gravitational force corresponds to the inertial force in classical mechanics.

\section{Summary and Discussions}

Through discussions in this paper and literature [32], we know that, if there is an inertial reference with no gravity, a non-trivial gravitational gauge field will be generated in the reference after a Lorentz transformation. In other words, if the reference is an inertial reference before transformation and the gravitational gauge field vanishes in it, the gravitational gauge field does not vanish after a Lorentz transformation. The gravitational gauge field not only affects the space-time structure, but also generates non-trivial gravitational force. The non-trivial gravitational force is generated from vacuum by a Lorentz transformation, so we call it vacuum gravitational force. The gravitational gauge field $C_{\mu}^{\alpha}(x)$ after Lorentz transformation is called vacuum gravitational field.

If the gravitational gauge transformation is global, the gravitational gauge field $C_{\mu}^{\alpha}(x)$ after gravitational gauge transformation is a constant, and the corresponding field strength of the gravitational gauge field vanishes. But if the gravitational gauge transformation is local, the gravitational gauge field $C_{\mu}^{\alpha}(x)$ after gravitational gauge transformation is not a constant but a function of space-time coordinate $x^{\mu}$, and the corresponding field strength of the gravitational gauge field does not vanish. From Equation (30), we could see that, if the gravitational field strength $F_{\mu \nu}^{\prime \alpha}(x)$ does not vanish, the gravitational force on a mass point does no vanish also. From the calculation in this paper, the force $f^{\mu}$ on the mass point $\mathrm{m}$ originates from gravitational gauge field $C_{\mu}^{\prime \alpha}(x)$. Therefore, this force is not a fictitious force. In other words, the vacuum gravitational force is not a fictitious force.

The correspondent of gravitational gauge transformation in classical mechanics is the transformation of a local reference. According to Equation (17), when the state of motion of a local reference is changed, the gravitational gauge field in that local reference will be changed accordingly. In other words, the gravitational gauge field in different local reference will be different, and the corresponding gravitational force on a mass point will also be different. The real gravitational force on a mass point depends on the state of motion of a reference. For example, an object which is at rest on the earth will feel the gravitational force of the earth. But if it is in a local inertial reference, it cannot feel any gravitational force, for the gravitational force of the earth and the vacuum gravitational force cancel each other out. This conclusion is the same as that in general relativity.

What is the classical correspondent of the vacuum gravitational force? According to the discussions in this paper, it is known that the magnitude of vacuum gravitational force is the mass times the acceleration of the reference. The direction of the vacuum gravitational force is just the opposite direction of the acceleration of the reference. These properties are the same as those of the inertial force in classical mechanics. Therefore, the classical correspondent of the vacuum gravitational force is the inertial 
force in classical mechanics.

Because the classical correspondent of the vacuum gravitational force is the inertial force, the nature of the inertial force is gravity. In other words, inertia and gravity are essentially the same, gravity and inertia are homologous, which is just the core idea of the equivalence principle of the general relativity. It is known that the equivalence principle is a transcendental principle in general relativity. But in quantum gauge theory of gravity, it is obtained through a strict derivation. Essentially speaking, it is only a deduction of the gauge principle.

Through the discussions in this paper, we know that there are two ways to produce gravitational field and gravitational force. One way is that it is produced by a massive object, which is given by classical Newtonian gravity and general relativity. Another way is that it is produced by a transformation, which is a new way to produce gravitational field and gravitational force. It is an inevitable outcome of gravitational gauge symmetry.

\section{References}

[1] Feynman, R.P., Leighton, R.B. and Sands, M.L. (2006) The Feynman Lectures on Physics. Pearson/Addison-Wesley, San Francisco.

[2] Born, M. and Leibfried, G. (1962) Einstein's Theory of Relativity. Courier Dover Publications, New York, 76-78.

[3] Einstein, A. (1923) The Principle of Relativity. Dover Publications, 111.

[4] Weinberg, S. (1972) Gravitation and Cosmology: Principles and Applications of the General Theory of Relativity. John Wiley \& Sons Ltd., New York.

[5] Straumann, N. (1984) General Relativity and Relativistic Astrophysics. Springer-Verlag, Berlin, Heidelberg, New York, Tokyo. http://dx.doi.org/10.1007/978-3-642-84439-3

[6] Wu, N. (2001) Gauge Theory of Gravity. http://arxiv.org/pdf/hep-th/0109145

[7] Wu, N. (2002) Communications in Theoretical Physics (Beijing, China), 38, 151-156.

[8] Wu, N. (2001) Quantum Gauge Theory of Gravity. http://arxiv.org/pdf/hep-th/0112062

[9] Wu, N. (2002) Quantum Gauge Theory of Gravity. Meeting of the Division of Particles and Fields of American Physical Society at the College of William \& Mary (DPF2002), Williamsburg, 24-28 May 2002. http://dpf2002.velopers.net/talks_pdf/33talk.pdf

[10] Wu, N. (2004) Communications in Theoretical Physics (Beijing, China), 42, 543-552.

[11] Wu, N. (2003) Renormalizable Quantum Gauge General Relativity. http://arxiv.org/pdf/gr-qc/0309041

[12] Wu, N. (2006) Focus on Quantum Gravity Research. In: Moore, D.C., Ed., Quantum Gauge Theory of Gravity, Nova Science Publishers, Inc., New York, 121-169.

[13] Wu, N. (2003) Communications in Theoretical Physics (Beijing, China), 40, 337-340.

[14] Wu, N. (2007) Communications in Theoretical Physics (Beijing, China), 47, 503-511.

[15] Wu, N. (2005) Communications in Theoretical Physics (Beijing, China), 44, 883-886. http://dx.doi.org/10.1088/6102/44/5/883

[16] Wu, N. (2007) Communications in Theoretical Physics (Beijing, China), 48, 469-472. http://dx.doi.org/10.1088/0253-6102/48/3/018

[17] Wu, N. (2008) Communications in Theoretical Physics (Beijing, China), 49, 129-132. 
http://dx.doi.org/10.1088/0253-6102/49/1/29

[18] Wu, N. (2008) Communications in Theoretical Physics (Beijing, China), 49, 1533-1540. http://dx.doi.org/10.1088/0253-6102/49/6/39

[19] Wu, N. (2004) Communications in Theoretical Physics (Beijing, China), 41, 567-572. http://dx.doi.org/10.1088/0253-6102/41/4/567

[20] Wu, N. (2006) Communications in Theoretical Physics (Beijing, China), 46, 639-642. http://dx.doi.org/10.1088/0253-6102/46/4/016

[21] Wu, N. (2006) Communications in Theoretical Physics (Beijing, China), 45, 452-456. http://dx.doi.org/10.1088/0253-6102/45/3/016

[22] Wu, N. and Zhang, D.-H. (2006) Communications in Theoretical Physics (Beijing, China), 45, 858-860. http://dx.doi.org/10.1088/0253-6102/45/5/017

[23] Wu, N. (2003) Communications in Theoretical Physics (Beijing, China), 40, 429-434. http://dx.doi.org/10.1088/0253-6102/40/4/429

[24] Wu, N. (2004) Communications in Theoretical Physics (Beijing, China), 41, 381-384. http://dx.doi.org/10.1088/0253-6102/41/3/381

[25] Wu, N. (2002) Communications in Theoretical Physics (Beijing, China), 38, 322-326. http://dx.doi.org/10.1088/0253-6102/38/3/322

[26] Wu, N. (2002) Communications in Theoretical Physics (Beijing, China), 38, 455-460. http://dx.doi.org/10.1088/0253-6102/38/4/455

[27] Wu, N. (2003) Communications in Theoretical Physics (Beijing, China), 39, 561-568.

[28] Wu, N. (2006) Chapter 3. Quantum Gravity Research Trends. In: Reimer, A., Ed., Unified Theory of Fundamental Interactions, Nova Science Publishers, Inc., New York, 83-122.

[29] Wu, N. (2001) Communications in Theoretical Physics (Beijing, China), 36, 169-172. http://dx.doi.org/10.1088/0253-6102/36/2/169

[30] Wu, N. (2002) Communications in Theoretical Physics (Beijing, China), 38, 577-582. http://dx.doi.org/10.1088/0253-6102/38/5/577

[31] Wu, N. (2003) Communications in Theoretical Physics (Beijing, China), 39, 671-674. http://dx.doi.org/10.1088/0253-6102/39/6/671

[32] Wu, N. (2014) Journal of Modern Physics, 5, 940-947. http://dx.doi.org/10.4236/jmp.2014.510097

[33] Wu, N. (2012) Gauge Gravity and Space-Time. http://arxiv.org/pdf/1207.5411 
Submit or recommend next manuscript to SCIRP and we will provide best service for you:

Accepting pre-submission inquiries through Email, Facebook, LinkedIn, Twitter, etc. A wide selection of journals (inclusive of 9 subjects, more than 200 journals)

Providing 24-hour high-quality service

User-friendly online submission system

Fair and swift peer-review system

Efficient typesetting and proofreading procedure

Display of the result of downloads and visits, as well as the number of cited articles

Maximum dissemination of your research work

Submit your manuscript at: http://papersubmission.scirp.org/

Or contact jmp@scirp.org 